\title{
A multicenter retrospective study of transurethral prostate split for benign prostate hyperplasia
}

Jingchao Wei ${ }^{1}$, Shigeng Zhang ${ }^{1}$, Bohan Wang ${ }^{1}$, Mang $\mathrm{Ke}^{2}$, Sheng Liu ${ }^{3}$, Zhengjia Yang ${ }^{4}$, Guoyun Zhou ${ }^{5}$, Jiacheng Qian ${ }^{6}$, Wenhui Lv ${ }^{7}$, Yi Fan ${ }^{8}$, Zhan Shi ${ }^{9}$, Lijun Wan ${ }^{10}$, Yongliang Chen ${ }^{11}$, Jinkui He ${ }^{12}$, Hui Liang ${ }^{13}$, Huimin Long $^{14}$, Shijian Wang ${ }^{15}$, Hao Wang ${ }^{16}$, Bing Chen ${ }^{17}$, Huan Shao ${ }^{18}$, Binbin Yang ${ }^{19}$, Chengfang Sun ${ }^{1}$, Qi Huangfu ${ }^{1}$, Chuanjun Du ${ }^{1}$, Ming Cai ${ }^{1}$, Jiaming Wen ${ }^{1}$

${ }^{1}$ Department of Urology, The Second Affiliated Hospital, School of Medicine, Zhejiang University, Hangzhou, China; ${ }^{2}$ Department of Urology, Taizhou Hospital of Zhejiang Province, Taizhou, China; ${ }^{3}$ Department of Urology, Taizhou Municipal Hospital, Taizhou, China; ${ }^{4}$ Department of Urology, Linping District Chinese Hospital, Hangzhou, China; ${ }^{5}$ Department of Urology, Lanxi Chinese Hospital, Jinhua, China; ${ }^{6}$ Department of Urology, Cixi Third People's Hospital, Ningbo, China; ${ }^{7}$ Department of Urology, Yongiia People's Hospital, Wenzhou, China; ${ }^{8}$ Department of Urology, Zhejiang Xiaoshan Hospital, Hangzhou, China; ${ }^{9}$ Department of Urology, Taizhou First People's Hospital, Taizhou, China; ${ }^{10}$ Department of Urology, Quzhou People's Hospital, Quzhou, China; ${ }^{11}$ Department of Urology, Shaoxing Central Hospital, Shaoxing, China; ${ }^{12}$ Department of Urology, Yiwu Chinese Hospital, Jinhua, China; ${ }^{13}$ Department of Urology, Xinchang County Hospital of Traditional Chinese Medicine, Shaoxing, China; ${ }^{14}$ Department of Urology, Ningbo Medical Center Li Huili Hospital, Ningbo, China; ${ }^{15}$ Department of Urology, Tongxiang First People's Hospital, Jiaxing, China; ${ }^{16}$ Department of Urology, Naval Hospital of Eastern Theater, Zhoushan, China; ${ }^{17}$ Department of Urology, Zhejiang Zhoushan Hospital, Zhoushan, China; ${ }^{18}$ Department of Urology, Jiaxing Chinese Hospital, Jiaxing, China; ${ }^{19}$ Department of Urology, Ningbo First Hospital, Ningbo, China

Contributions: (I) Conception and design: J Wei, J Wen; (II) Administrative support: J Wen; (III) Provision of study materials or patients: All authors; (IV) Collection and assembly of data: All authors; (V) Data analysis and interpretation: J Wei, J Wen; (VI) Manuscript writing: All authors; (VII) Final approval of manuscript: All authors.

Correspondence to: Jiaming Wen. Department of Urology, The Second Affiliated Hospital, School of Medicine, Zhejiang University, Hangzhou, China. Email: wenjiaming@zju.edu.cn.

Background: Transurethral split of the prostate (TUSP) is effective in treating benign prostatic hyperplasia (BPH). However, there is still a lack of research focusing on the optimal target population for TUSP. This study aimed to compare the efficacy of TUSP in patients with different prostate volumes or ages.

Methods: The study was a multicenter retrospective study. The outcomes of TUSP in BPH patients with different prostate volumes or different ages were compared. A total of 439 patients were included in the study. Patients were divided into two groups according to prostate volume, with a cut-off value of $50 \mathrm{~mL}$. Similarly, the cut-off value for the age groups was 70 years. Baseline patient characteristics and perioperative outcomes were recorded. Follow-up was performed at 1, 6, and 12 months after surgery.

Results: The mean age of the patients was 73.4 years, and the mean prostate volume was $51.2 \mathrm{~mL}$. At 12-month follow-up after TUSP treatment, the patients' International Prostate Symptom Scores (IPSS), quality of life (QoL) scores, and postvoid residual (PVR) volumes decreased significantly, while peak urinary flow rate (Qmax) increased significantly. Intraoperative hemoglobin $(\mathrm{Hb})$ reduction was significantly lower in the small volume group than in the large volume group. The incidence of postoperative urinary urgency and transient incontinence was lower in the small volume group. IPSS score, PVR, and Qmax in the small volume group showed more remarkable changes at several time points compared to the preoperative period. Postoperative pain scores were higher in the small volume group than in the large volume group. There were no differences between the two groups in terms of long-term complications. The younger group showed greater variation in PVR and Qmax at some time points but less variation in QoL than the older group.

^ ORCID: 0000-0002-1037-7990. 


\begin{abstract}
Conclusions: TUSP is overall safe and effective in treating BPH. This study showed differences in the outcomes of TUSP in treating different prostate volumes or ages of BPH patients. The optimal surgical approach for $\mathrm{BPH}$ patients might be selected clinically based on a combination of prostate volume or patient age.
\end{abstract}

Keywords: Benign prostatic hyperplasia (BPH); transurethral split of the prostate (TUSP); prostate volume; age

Submitted Nov 29, 2021. Accepted for publication Jan 30, 2022.

doi: $10.21037 / \mathrm{tau}-21-1138$

View this article at: https://dx.doi.org/10.21037/tau-21-1138

\section{Introduction}

Benign prostatic hyperplasia $(\mathrm{BPH})$ is prevalent in middleaged and older men and has an escalating incidence with increasing age (1). The prostate gland is located at the outlet of the bladder and is an essential organ for controlling urine flow in males. Due to the uniqueness of its anatomical location, the enlarged prostate compresses inwards centrally and causes urinary tract obstruction, leading to dysuria and a series of lower urinary tract symptoms (LUTS), such as micturition frequency, micturition urgency, and micturition pain (2). The main risk factors for the development of BPH include: advanced age; family history of $\mathrm{BPH}$; physical conditions such as obesity, heart and circulatory disease, and type 2 diabetes mellitus; and erectile dysfunction. It is noteworthy that, if treated improperly or untimely, $\mathrm{BPH}$ will gradually progress and worsen, with urine reflux eventually complicating upper urinary tract fluid accumulation and affecting both kidneys (3).

Fundamental research has developed various strategies to treat $\mathrm{BPH}$ in the past few decades, mainly focusing on medication and surgery (4). If the symptoms of LUTS caused by BPH are moderate, close observation may be an optimal choice. Medications used to treat BPH may have side effects, including low blood pressure, dizziness, and nausea. In severe cases of LUTS, medications such as alpha-adrenergic blockers and 5-alpha reductase inhibitors are available. If recurrent urinary retention, complicated urinary tract infections, bladder stones, renal insufficiency, or persistent hematuria occur despite drug treatment, these suggest that drug treatment has failed, and surgical treatment should be chosen (5). There are currently several surgical approaches for BPH. Transurethral resection of the prostate (TURP) has been used clinically for many years and serves as the accepted treatment option for BPH with surgical indications (6). While TURP provides an overarching favorable surgical benefit, it is comparatively invasive, and there are potential risks, as with all types of surgery. Complications after surgery may include urinary tract infections, hematuria, dysuria, urinary incontinence, and sexual dysfunction. The incidence of retrograde ejaculation after surgery is as high as $90 \%$ (7). Transient incontinence is prevalent and can persist for a few weeks after the operation, but occasionally it can become longterm incontinence. Up to $10 \%$ of men have difficulty obtaining and maintaining a satisfactory erection after surgery (8). During the TURP procedure, thermal or mechanical damage may cause injury to the bladder neck and postoperative bladder neck contracture (BNC). The overall incidence of post-TURP BNC was reported around $5.4 \%$, mostly at 6 months postoperatively (9). Furthermore, the prostate gland is partially excised during TURP, which results in a comparatively prolonged postoperative hospital stay and higher overall medical costs. Consequently, patients with $\mathrm{BPH}$ are required to have good physical and financial conditions to be suitable for TURP (10).

Given the potential risks associated with TURP, several less invasive operations have been developed to treat BPH. Holmium laser enucleation of the prostate (HoLEP) is one of the most representative of these techniques (11). The EAU 2019 guidelines recommend HoLEP as a firstline treatment option for sizeable prostatic hyperplasia $(>80 \mathrm{~mL})(12)$. For small volume $\mathrm{BPH}$, the prostate envelope is not sufficiently clear, and performing HoLEP increases the risk of injury (13). In addition, although the guidelines recommend transurethral incision of the prostate (TUIP) as the first-line treatment for small volume prostates without a middle lobe, some patients with a small volume prostate have a prominent middle lobe (14). Other frequently applied surgical approaches include bipolar transurethral plasma kinetic prostatectomy (TUPKP) and transurethral plasma kinetic enucleation of the prostate 
(TUKEP) $(15,16)$. Nevertheless, the equipment needed for these procedures is relatively expensive, and the learning curve for surgeons is rather long, leading to a disincentive for the clinical use of these operations in primary care. Transurethral dilation of the prostate (TUDP) was popular decades ago. TUDP is simple to master and less invasive. However, subsequent clinical practice has revealed several disadvantages of TUDP, mainly regarding the uncertainty of its efficacy (17). Its smaller invasiveness is often associated with the risk of recurrence or re-operation.

Transurethral split of the prostate (TUSP) is an improvement on TUDP, mainly by replacing the spherical balloon with a double-column balloon catheter (18). TUSP dilates the prostatic urethra and bladder neck simultaneously with the aid of a double-column balloon catheter (19). Since TUSP dilates the prostatic envelope at the 12 o'clock position of the prostatic apex with the help of an internal balloon, it effectively reduces the closing pressure of the urethra. The external balloon then expands, dilating the urethra and reducing the tension on the urethral wall (1). As a new method for treating BPH, TUSP can improve urinary function without compromising the sexual and reproductive function of the prostate. A clinical study involving 113 patients showed that the column balloon catheter efficaciously treated BPH (19). TUSP preserves the prostate gland and allows dilation of the obstructed prostatic urethra in a simple and minimally invasive manner. It is thus particularly suitable for patients who need to preserve their sexual function, have multiple underlying diseases, have poor anesthetic tolerance, or are older. However, a more comprehensive and exhaustive evaluation is missing due to the small number of relevant studies.

The mechanisms by which BPH contributes to LUTS symptoms involve three cardinal aspects: enlarged prostate volume, smooth muscle contraction, and fibrosis (20). For $\mathrm{BPH}$ with a relatively larger prostate, the prostate volume is the leading cause of LUTS. In contrast, prostate fibrosis and altered smooth muscle contraction are primarily responsible for LUTS in comparatively smaller prostate glands (21). Owing to the differences in the principal mechanisms that contribute to LUTS, it is interesting to investigate the effectiveness of TUSP in treating BPH with different prostate volumes. This multicenter study retrospectively collected data from patients who underwent TUSP. Although a comprehensive analysis of $565 \mathrm{BPH}$ patients who underwent TUSP was previously conducted by Huang et al., their study primarily evaluated the long-term outcomes of
TUSP for treating BPH (18). In contrast, our study mainly compared $\mathrm{BPH}$ patients of different ages or different prostate volumes with the aim of exploring the optimal population for TUSP. Elderly patients often have comorbidities that make treatments like TURP challenging to endure. Besides, elderly BPH patients tend to have a longer course and may have more remarkable structural changes in the prostate. Therefore, it is important to study the effect of age on TUSP outcomes. Several studies have shown that $70 \%$ of men over 70 have pathological BPH (22). In short, this multicenter retrospective study aimed to comparatively evaluate the effectiveness of TUSP in treating BPH patients with different prostate volumes or different ages. We present the following article in accordance with the STROBE reporting checklist (available at https://tau.amegroups.com/article/ view/10.21037/tau-21-1138/rc).

\section{Methods}

\section{Patients}

The study was approved by institutional ethics board of The Second Affiliated Hospital, School of Medicine, Zhejiang University (No. 2021-1062). All procedures performed in studies involving human participants were in accordance with the ethical standards of the institutional and/or national research committee(s) and with the Helsinki Declaration (as revised in 2013). Written informed consents were obtained from all patients. A total of 439 patients who underwent TUSP from September 2015 to August 2020 were enrolled in this study. These patients were collected from multiple centers and had no history of other surgical prostate procedures. Indications for BPH surgery included failure of drug therapy, refractory urinary retention, recurrent urinary tract infections, persistent hematuria, bladder stones, and renal insufficiency. Patients with no follow-up data or those who refused to join the study were excluded.

\section{Diagnosis of $\mathrm{BPH}$}

The diagnosis of BPH depends on the histological proliferation of the interstitial and glandular components of the prostate, the anatomical enlargement of the prostate, the clinical symptoms dominated by lower urinary tract symptoms, and the urodynamic obstruction of the bladder outlet. A combination of symptoms, physical examination especially rectal examinations, imaging, urodynamics and 
Table 1 Baseline patient characteristics

\begin{tabular}{lc}
\hline Parameters & Descriptive $(\mathrm{n}=439)$ \\
\hline Age (year), mean $\pm \mathrm{SD}$ & $73.4 \pm 10.8$ \\
$\mathrm{BMI}$, mean $\pm \mathrm{SD}$ & $20.8 \pm 2.7$ \\
$\mathrm{Hb}$ (preoperative) (g/L), mean $\pm \mathrm{SD}$ & $141.2 \pm 10.9$ \\
Hypertension, $\mathrm{n}$ & 129 \\
Diabetes mellitus, $\mathrm{n}$ & 110 \\
Cardiac insufficiency, $\mathrm{n}$ & 95 \\
Pulmonary insufficiency, $\mathrm{n}$ & 62 \\
Cerebrovascular function sequelae, $\mathrm{n}$ & 38 \\
\hline
\end{tabular}

$\mathrm{SD}$, standard deviation; BMI, body mass index, $\mathrm{Hb}$, hemoglobin.

endoscopy can effectively diagnose BPH.

\section{Data collection}

Baseline demographic factors including age and body mass index (BMI) were gathered. BMI is calculated by the patient's weight in kilograms divided by their height in meters squared. Any history of underlying medical conditions such as hypertension, diabetes, cardiac insufficiency, and pulmonary insufficiency was also collected. Prostate volume was measured by transrectal ultrasound before surgery and calculated by leveraging the formula length $x$ width $\times$ height $\times \pi / 6$. Urodynamic examinations were used to obtain peak urinary flow rate (Qmax) values, and ultrasonography was used to determine postvoid residual (PVR) volumes. The International Prostate Symptom Score (IPSS) and quality of life (QoL) scores were acquired through the IPSS and QoL scales, respectively. Qmax, PVR, IPSS, and QoL were measured preoperatively and at 1, 6, and 12 months after surgery. Hemoglobin $(\mathrm{Hb})$ was detected preoperatively and on the second day postoperatively. Operative time and several perioperative measurement factors, including catheterization time, postoperative hospital stay, pain scores, and transurethral resection syndrome (TURS) were also gathered. The occurrence of postoperative bleeding, micturition frequency, micturition urgency, micturition pain, urinary tract infection, re-catheterization, transient incontinence, late complications, BNC, and continuous incontinence was followed up for 12 months.

\section{Basis of grouping}

The mean preoperative prostate volume of all patients in this study was approximately $50 \mathrm{~mL}$, so we chose $50 \mathrm{~mL}$ as the cut-off value to compare the efficacy of TUSP between large and small prostate volumes. The mean age of all patients in this study was also close to 70 years. Therefore, 70 years was chosen as the cut-off value for the age comparison in this study.

\section{Operative procedure}

The operative procedure for TUSP has been described previously (21).

\section{Statistical analysis}

Continuous values were presented as mean \pm standard deviation (SD). The Shapiro-Wilk normality test was performed for continuous variables. The independent samples $t$-test was used to compare the means of the two groups. For discrete variables, chi-square tests were conducted for comparisons between groups. Pearson's chi-square was used if all frequencies were $\geq 5$, and Yates' corrected chi-square was used if any frequency occurred $<5$. The Statistical Package for the Social Sciences version 22.0 (SPSS, Chicago, IL, USA) was used for the statistical analysis. $P$ values $<0.05$ were considered statistically significant.

\section{Results}

\section{Description of patients' preoperative characteristics}

A total of 439 patients who underwent TUSP surgery were included. The mean age of the patients was $73.4 \pm 10.8$ years, the mean BMI was $20.8 \pm 2.7$, and the mean preoperative $\mathrm{Hb}$ was $141.2 \pm 10.9 \mathrm{~g} / \mathrm{L}$. As prostatic hyperplasia patients tend to be middle-aged and older men, the patients often present with some underlying diseases. We collected information on the patients' hypertension, diabetes mellitus, cardiac insufficiency, and pulmonary insufficiency. The results showed 129 cases of hypertension, 110 cases of diabetes mellitus, 95 cases of cardiac insufficiency, 62 cases of pulmonary insufficiency, and 38 cases of cerebrovascular function sequelae (Table 1).

In addition, the prostate-related specialist profile was assessed in all patients before surgery. The mean volume of the prostate measured by preoperative ultrasound was $51.2 \pm 33.2 \mathrm{~mL}$. The mean value of the prostate volume in this study was also an essential basis for selecting $50 \mathrm{~mL}$ as the threshold value for grouping patients. The mean PSA was $1.7 \pm 1.3 \mathrm{ng} / \mathrm{mL}$. The mean IPSS score was $27.6 \pm 3.6$, and the mean QoL score was $5.4 \pm 0.8$, indicating that $\mathrm{BPH}$ 
Table 2 Preoperative prostate-related parameters

\begin{tabular}{lc}
\hline Parameters & Descriptive $(\mathrm{n}=439)$, mean $\pm \mathrm{SD}$ \\
\hline Prostate volume $(\mathrm{mL})$ & $51.2 \pm 33.2$ \\
PSA $(\mathrm{ng} / \mathrm{mL})$ & $1.7 \pm 1.3$ \\
IPSS score & $27.6 \pm 3.6$ \\
QoL score & $5.4 \pm 0.8$ \\
PVR $(\mathrm{mL})$ & $56.8 \pm 7.9$ \\
Qmax $(\mathrm{mL} / \mathrm{s})$ & $7.6 \pm 1.7$
\end{tabular}

SD, standard deviation; PSA, prostate-specific antigen; IPSS, International Prostate Symptom Score; QoL, quality of life; PVR, postvoid residual volume; Qmax, peak urinary flow rate.

severely impacts patients' QoL. Furthermore, the mean preoperative PVR for all patients was $56.8 \pm 7.9 \mathrm{~mL}$, and the mean Qmax was $7.6 \pm 1.7 \mathrm{~mL} / \mathrm{s}$, indicating that patients were severely impaired in urination (Table 2).

\section{The overall outcome of TUSP}

The IPSS score, QoL score, PVR, and Qmax were collected at four different time points: preoperative, and 1, 6, and 12 months postoperative. The total number of cases for which complete data were collected at the four time points were 439, 434, 431, and 427. The IPSS and QoL scales are well established and reliable determinants of the level of distress or effectiveness of treatment for men with LUTS. The mean IPSS score was 27.6 \pm 3.6 , representing severe LUTS, and the mean QoL score was 5.5 \pm 0.8 , representing patients' distress. The data showed significant amelioration of LUTS and significant improvement in QoL in the 12 months following the TUSP procedure. The mean preoperative PVR was $56.7 \pm 7.9 \mathrm{~mL}$, and the mean Qmax was $7.6 \pm 1.7 \mathrm{~mL} / \mathrm{s}$. PVR was significantly lower at the three postoperative time points compared with the preoperative level and showed a gradual decrease over time. Qmax was significantly higher at all postoperative time points than preoperatively and increased progressively over time. The 12 -month postoperative follow-up data suggested that TUSP effectively treated BPH and significantly alleviated dysuria (Table 3).

\section{Comparison between different prostate volumes}

To compare the efficacy of TUSP in treating BPH patients with different prostate volumes, we divided all patients into two groups based on a cut-off value of $50 \mathrm{~mL}$, as described previously. Patients in the large volume prostate group were significantly older $(75.6 \pm 11$ years $)$ than those in the small volume prostate group $(71.9 \pm 10.5)$ and had a significantly lower BMI than the small volume group $(20.2 \pm 2.7 \mathrm{vs}$. $21.3 \pm 2.7$, respectively). There was no significant difference in the preoperative $\mathrm{Hb}$ levels between the two groups. In terms of underlying disease, there were no differences in hypertension, diabetes mellitus, cardiac insufficiency, pulmonary insufficiency, or cerebrovascular function sequelae between the two groups (Table 4).

To investigate the differences in the therapeutic risk of TUSP for BPH with different prostate volumes, we collected perioperative data from patients. Analysis of the perioperative observations in both groups revealed that TUSP reduced $\mathrm{Hb}$ by $6.9 \pm 2.3 \mathrm{~g} / \mathrm{L}$ in the large volume prostate group, which was significantly more than the $6.1 \pm 2.3 \mathrm{~g} / \mathrm{L}$ in the small volume group. Overall, intraoperative blood loss was minimal in both groups but less in the small volume prostate group. There were no differences between the two groups regarding operative time, catheterization time, postoperative hospital stay, or postoperative pain scores. No TURS was recorded for any patients (Figure 1A-1E).

During the 12-month follow-up period, 427 patients were successfully followed up. The complications were divided into early and late complications according to the time of their onset. Micturition urgency was significantly higher in the large volume prostate group than in the small volume group $(\mathrm{P}=0.016)$. Transient urinary incontinence occurred at a significantly lower rate in the small volume group than in the large volume group $(\mathrm{P}=0.007)$. There was no difference between the two groups in postoperative bleeding, micturition frequency, micturition urgency, micturition pain, urinary tract infection, and recatheterization. In terms of long-term complications, the incidence of BNC was low in all cases and did not differ between the groups. No continuous incontinence was recorded in any of the patients (Table 5).

At the 12-month follow-up, we compared the degree of change in the IPSS scores, QoL scores, PVR, and Qmax, using the preoperative observation level as the baseline between the two groups. At all three postoperative time points, the degree of change in the IPSS score was greater in the small volume group than in the large volume group. The degree of QoL change was not significantly different between the two groups. The change in PVR was significantly greater in the small volume group than in the large volume group. There was no difference in 
Table 3 The overall outcome of TUSP

\begin{tabular}{|c|c|c|c|c|}
\hline Parameters & $\begin{array}{l}\text { Pre-operation } \\
\qquad(\mathrm{n}=439)\end{array}$ & $\begin{array}{l}1 \text { month post-operation } \\
\qquad(\mathrm{n}=434)\end{array}$ & $\begin{array}{l}6 \text { months post-operation } \\
\qquad(\mathrm{n}=431)\end{array}$ & $\begin{array}{l}12 \text { months post-operation } \\
\qquad(\mathrm{n}=427)\end{array}$ \\
\hline IPSS score, mean \pm SD & $27.6 \pm 3.6$ & $10.3 \pm 3.5$ & $6.8 \pm 3.3$ & $7.1 \pm 2.9$ \\
\hline QoL score, mean \pm SD & $5.5 \pm 0.8$ & $2 \pm 0.9$ & $1.5 \pm 0.7$ & $1.4 \pm 0.7$ \\
\hline$P$ & & $<0.001^{\star \star \star}$ & $<0.001^{\star \star \star}$ & $<0.001^{\star \star \star}$ \\
\hline $\mathrm{Qmax}(\mathrm{mL} / \mathrm{s})$, mean $\pm \mathrm{SD}$ & $7.6 \pm 1.7$ & $16.7 \pm 3.2$ & $18.7 \pm 3$ & $18.6 \pm 2.9$ \\
\hline $\mathrm{P}$ & & $<0.001^{\star \star \star}$ & $<0.001^{\star \star \star}$ & $<0.001^{\star \star \star}$ \\
\hline
\end{tabular}

${ }^{\star * \star}, \mathrm{P}<0.001$. TUSP, transurethral split of the prostate; IPSS, International Prostate Symptom Score; QoL, quality of life; PVR, postvoid residual volume; Qmax, peak urinary flow rate.

Table 4 Baseline characteristics of patients with different prostate volumes

\begin{tabular}{|c|c|c|c|}
\hline \multirow{2}{*}{ Parameters } & \multicolumn{2}{|c|}{ Prostate volume } & \multirow{2}{*}{$\mathrm{P}$} \\
\hline & $\leq 50 \mathrm{~mL}(\mathrm{n}=262)$ & $>50 \mathrm{~mL}(\mathrm{n}=177)$ & \\
\hline Age (year), mean $\pm S D$ & $71.9 \pm 10.5$ & $75.6 \pm 11$ & $<0.001^{\star \star *}$ \\
\hline BMI, mean \pm SD & $21.3 \pm 2.7$ & $20.2 \pm 2.7$ & $<0.001^{\star \star \star}$ \\
\hline $\mathrm{Hb}$ (preoperative) $(\mathrm{g} / \mathrm{L})$, mean $\pm \mathrm{SD}$ & $140.9 \pm 11.1$ & $141.7 \pm 10.7$ & 0.439 \\
\hline Diabetes mellitus, $\mathrm{n}$ & 60 & 50 & 0.205 \\
\hline Cardiac insufficiency, $\mathrm{n}$ & 59 & 36 & 0.586 \\
\hline Pulmonary insufficiency, $\mathrm{n}$ & 36 & 26 & 0.779 \\
\hline Cerebrovascular function sequelae, $\mathrm{n}$ & 24 & 14 & 0.648 \\
\hline
\end{tabular}

${ }^{* * *}, \mathrm{P}<0.001$. SD, standard deviation; BMI, body mass index, $\mathrm{Hb}$, hemoglobin.

the degree of change in Qmax between the two groups at 1 month postoperatively but was greater in the small volume group than in the large volume group at 6 and 12 months postoperatively (Figure $2 A-2 D$ ).

\section{Comparison between different ages}

To compare the TUSP outcome in BPH patients of different ages, we divided the patients into two groups using 70 years as the cut-off value. The comparative analysis of the baseline data showed that prostate volume was significantly larger in the older patient group than in the younger patient group, consistent with the general pattern of larger prostate sizes in older patients. BMI was significantly lower in the older patient group than in the younger group. There was also no difference in preoperative $\mathrm{Hb}$ levels between the two groups. For underlying diseases, hypertension and diabetes mellitus were significantly higher in the older group than in the younger group, consistent with the general observation that these diseases are more prevalent in the elderly. There was no difference in the incidence of cardiac insufficiency, pulmonary insufficiency, or cerebrovascular function sequelae between the two groups (Table 6).

We analyzed the observations during the perioperative period in both groups of patients. The results showed that $\mathrm{Hb}$ reduction was significantly greater in the older patient group than in the younger group. The postoperative pain 
A

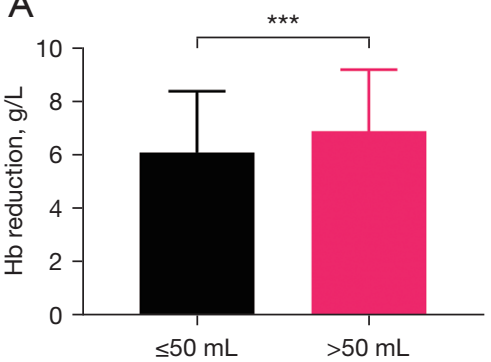

D

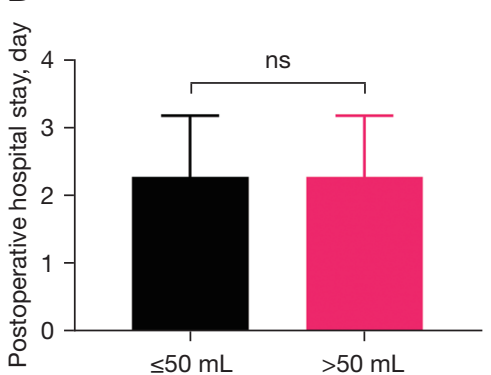

B

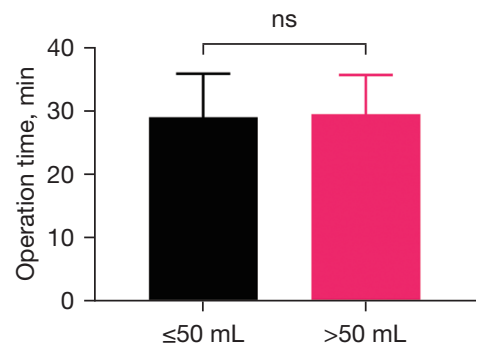

$\mathrm{E}$

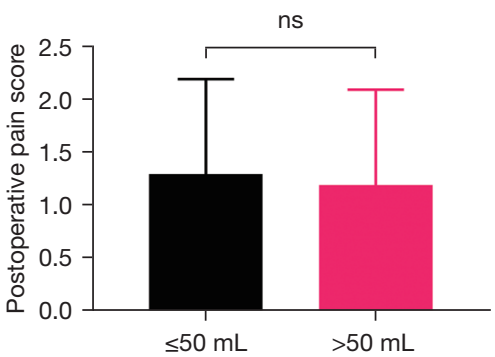

C

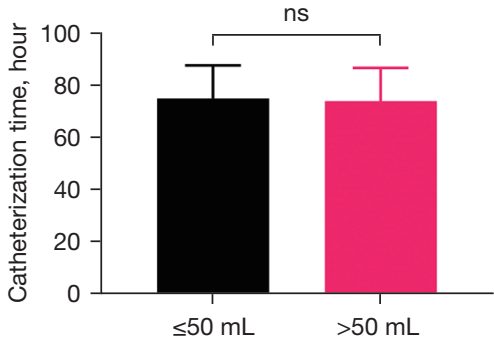

Figure 1 Perioperative parameters of patients with different prostate volumes. (A-E) Bar charts indicating Hb reduction, operation time, catheterization time, postoperative hospital stay, and postoperative pain scores, respectively. The cut-off value for volume grouping is $50 \mathrm{~mL} .{ }^{* * *}, \mathrm{P}<0.001 ;$ ns, not significant. Hb, hemoglobin.

Table 5 Follow-up data of patients with different prostate volumes

\begin{tabular}{|c|c|c|c|}
\hline Parameters & \multicolumn{2}{|c|}{ Prostate volume } & $P$ \\
\hline \multicolumn{4}{|l|}{ Early complications, $\mathrm{n}$} \\
\hline Postoperative bleeding & 2 & 4 & 0.217 \\
\hline Micturition frequency & 35 & 27 & 0.464 \\
\hline Micturition pain & 32 & 16 & 0.366 \\
\hline Urinary tract infection & 21 & 18 & 0.361 \\
\hline Re-catheterization & 10 & 9 & 0.464 \\
\hline Transient incontinence & 21 & 28 & $0.007^{\star \star}$ \\
\hline Continuous incontinence ( 1 year) & 0 & 0 & 1 \\
\hline
\end{tabular}

*, $\mathrm{P}<0.05$; ${ }^{* *}, \mathrm{P}<0.01$. BNC, bladder neck contracture.

score was significantly lower in the older patient group than the younger group. There were no differences between the two groups regarding operative time, catheterization time, or postoperative hospital stay (Figure $3 A-3 E$ ).
We then analyzed the postoperative complications between $\mathrm{BPH}$ patients of different ages. As previously mentioned, the follow-up period was 12 months. There was no difference between the two groups in postoperative bleeding, 
A

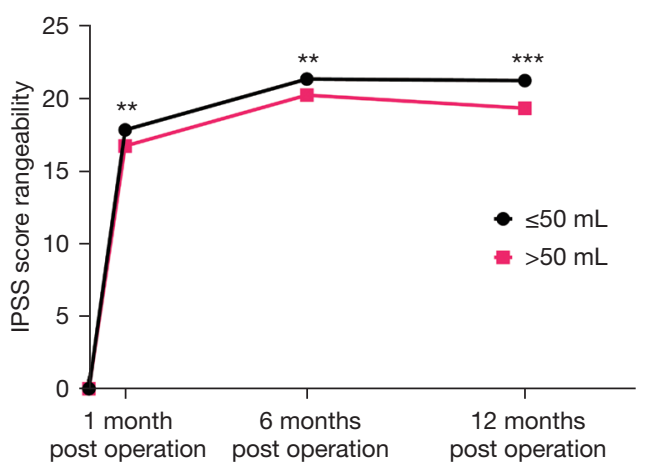

C

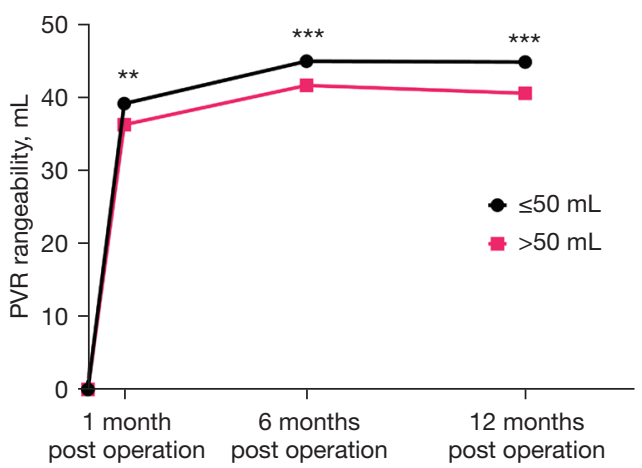

B

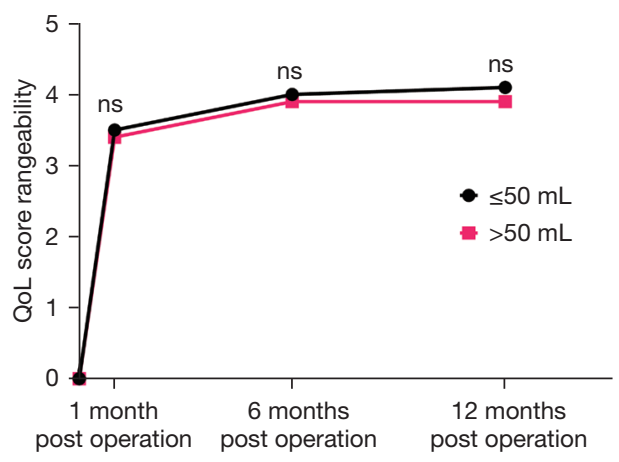

D

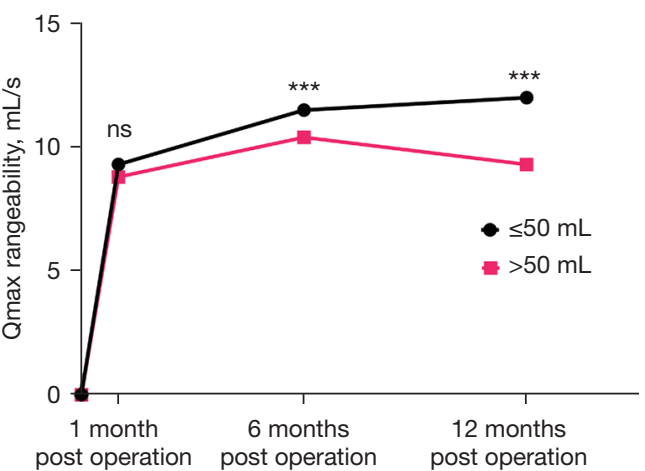

Figure 2 Comparison of TUSP outcome between patients with different prostate volumes. (A-D) The degree of change in IPSS scores, QoL scores, PVR, and Qmax, respectively, at 1, 6, and 12 months postoperatively. The cut-off value for volume grouping is $50 \mathrm{~mL} .{ }^{* *}$, $\mathrm{P}<0.01$; ${ }^{* *}, \mathrm{P}<0.001$; ns, not significant. TUSP, transurethral split of the prostate; IPSS, International Prostate Symptom Score; QoL, quality of life; PVR, postvoid residual volume; Qmax, peak urinary flow rate.

Table 6 Baseline characteristics for patients of different ages

\begin{tabular}{|c|c|c|c|}
\hline Parameters & \multicolumn{2}{|c|}{ Age } & $\mathrm{P}$ \\
\hline Prostate volume $(\mathrm{mL})$, mean $\pm \mathrm{SD}$ & $45.1 \pm 28.3$ & $54.7 \pm 35.3$ & $0.004^{\star \star}$ \\
\hline $\mathrm{BMI}$, mean $\pm \mathrm{SD}$ & $21.9 \pm 2.7$ & $20.2 \pm 2.6$ & $<0.001^{\star \star \star}$ \\
\hline $\mathrm{Hb}$ (preoperative) (g/L), mean $\pm \mathrm{SD}$ & $140.1 \pm 10.7$ & $141.9 \pm 11$ & 0.109 \\
\hline Diabetes mellitus, $\mathrm{n}$ & 30 & 80 & $0.021^{*}$ \\
\hline Cardiac insufficiency, $\mathrm{n}$ & 23 & 72 & $0.005^{\star \star}$ \\
\hline Pulmonary insufficiency, $\mathrm{n}$ & 22 & 40 & 0.865 \\
\hline Cerebrovascular function sequelae, $\mathrm{n}$ & 11 & 27 & 0.315 \\
\hline
\end{tabular}

*, $\mathrm{P}<0.05 ;{ }^{* \star}, \mathrm{P}<0.01 ;{ }^{* \star \star}, \mathrm{P}<0.001$. SD, standard deviation; BMI, body mass index, $\mathrm{Hb}$, hemoglobin. 
A

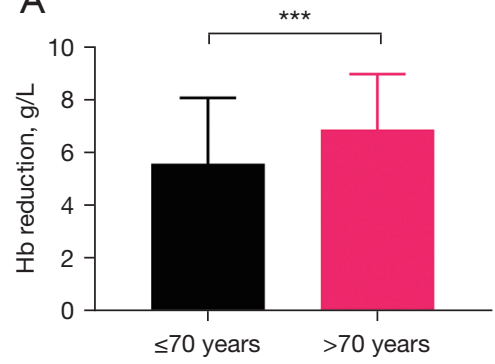

D

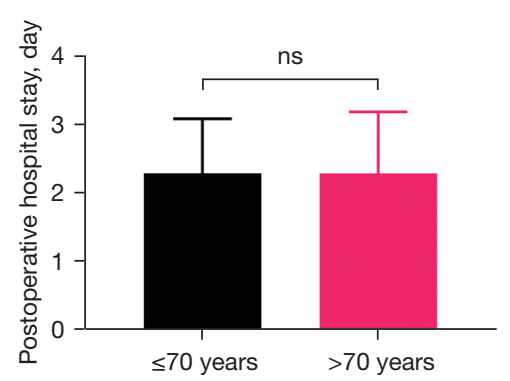

B

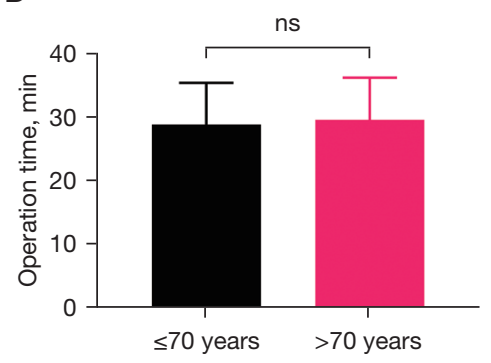

$E$

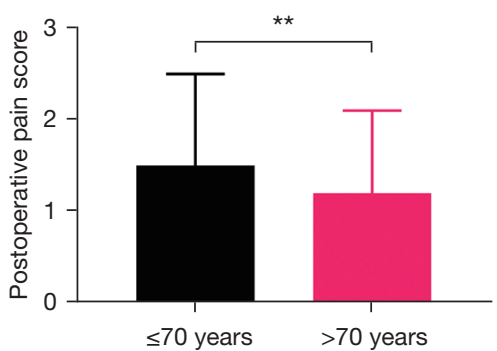

C

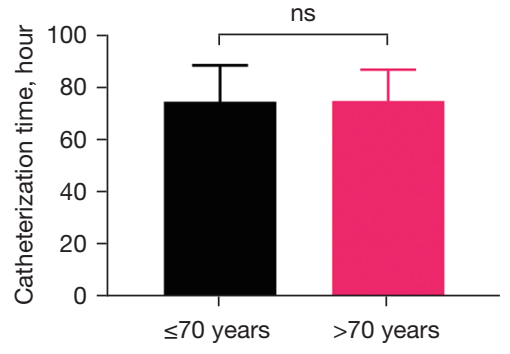

Figure 3 Perioperative parameters of patients of different ages. (A-E) Bar charts indicating Hb reduction, operation time, catheterization time, postoperative hospital stay, and postoperative pain score, respectively. The cut-off value for age grouping is $70 .{ }^{* *}, \mathrm{P}<0.01$; ${ }^{* * *}, \mathrm{P}<0.001 ;$ ns, not significant. $\mathrm{Hb}$, hemoglobin.

Table 7 Follow-up data for patients of different ages

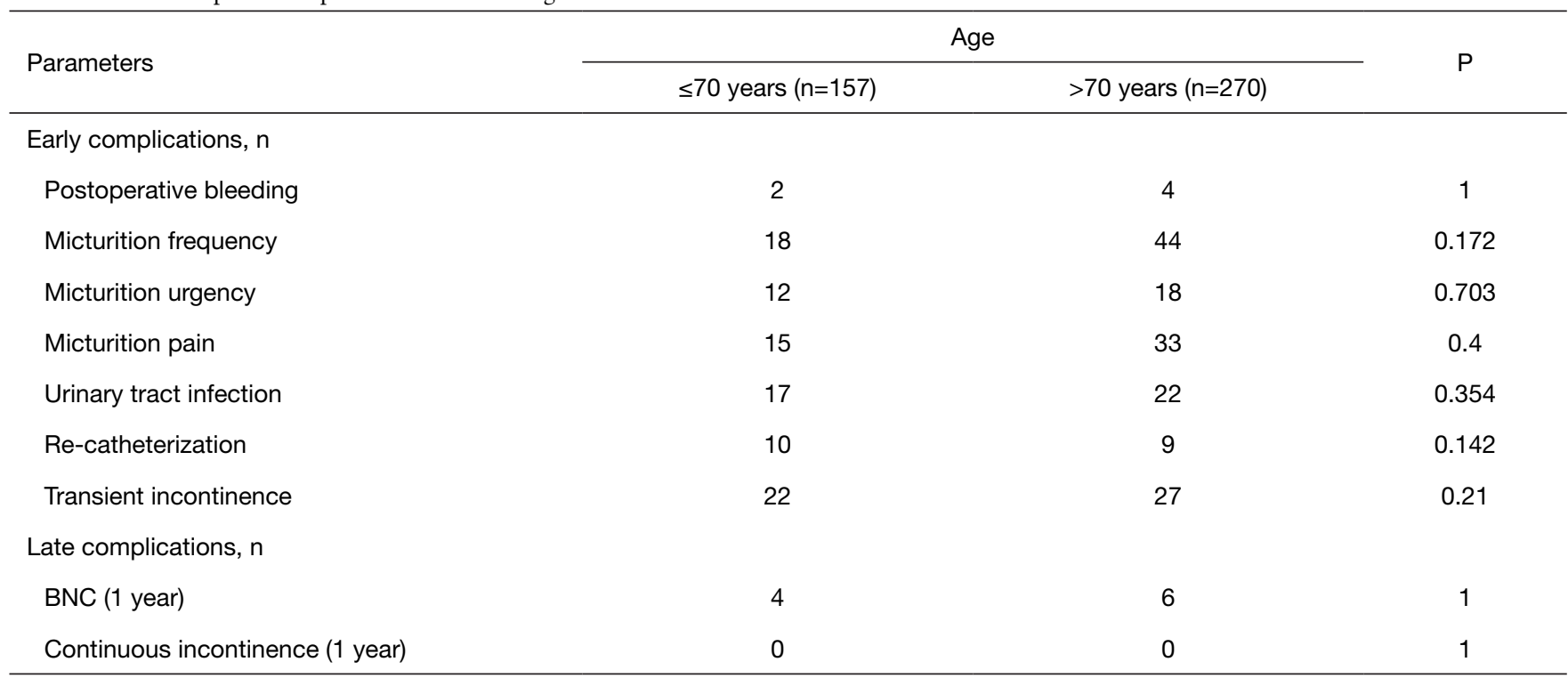

BNC, bladder neck contracture.

micturition frequency, micturition urgency, micturition pain, urinary tract infection, re-catheterization, transient urinary incontinence, or postoperative complications. In terms of long-term complications, there was also no difference between the two groups in BNC (12 months) and continuous incontinence (12 months) (Table 7).

Similarly, we compared the degree of change in IPSS scores, QoL scores, PVR, and Qmax between the 
A

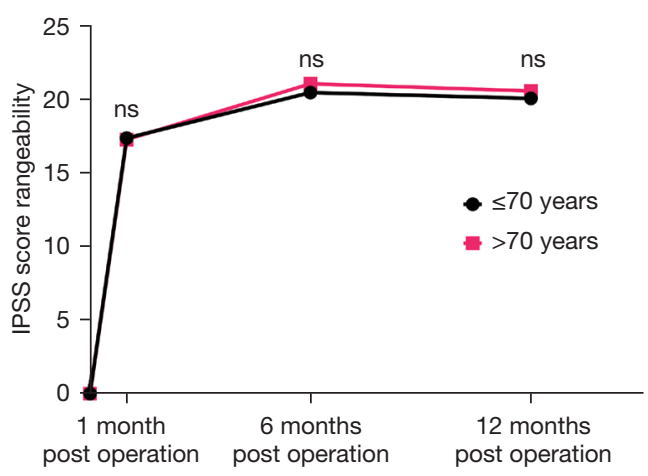

C

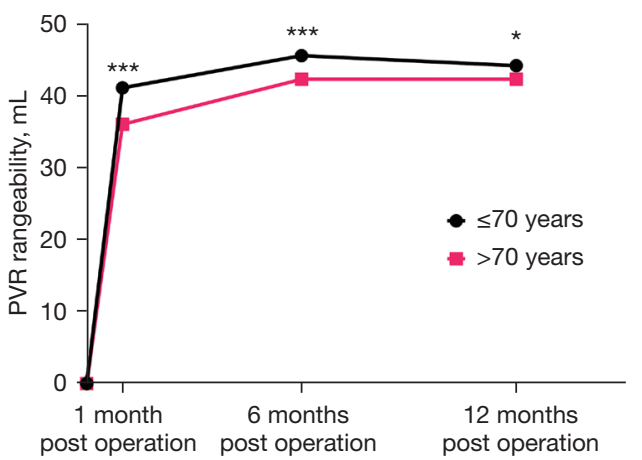

B

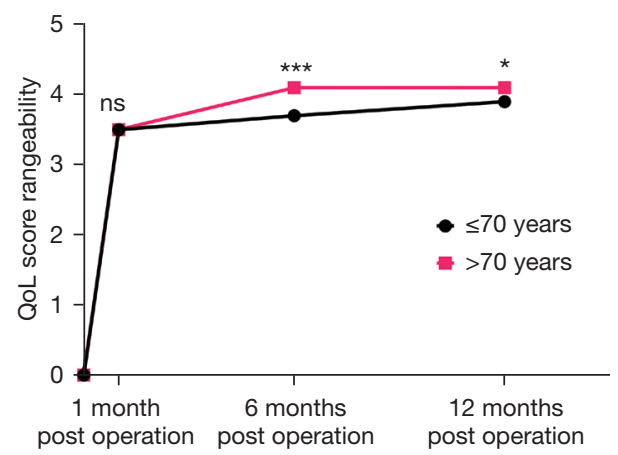

D

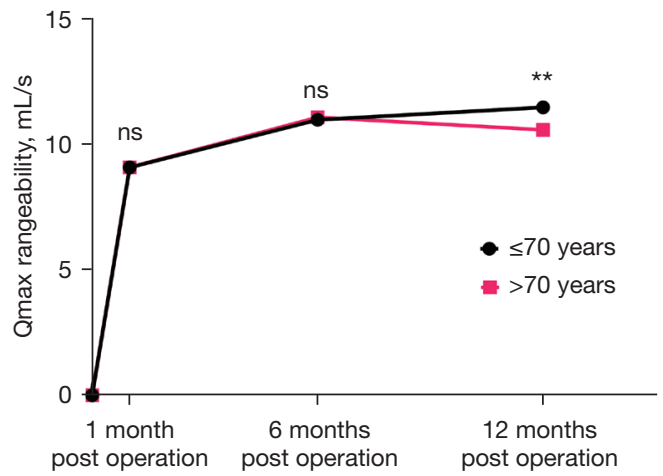

Figure 4 Comparison of TUSP outcome between patients of different ages. (A-D) The degree of change in IPSS scores, QoL scores, PVR, and Qmax, respectively, at 1, 6, and 12 months postoperatively. The cut-off value for age grouping is $70 .{ }^{*}, \mathrm{P}<0.05 ;{ }^{* *}, \mathrm{P}<0.01 ;{ }^{* * *}, \mathrm{P}<0.001$; ns, not significant. TUSP, transurethral split of the prostate; IPSS, International Prostate Symptom Score; QoL, quality of life; PVR, postvoid residual volume; Qmax, peak urinary flow rate.

two groups using the preoperative observation level as the baseline. There was no difference in the degree of change in IPSS scores between the two groups at the three postoperative time points. The change in QoL at 6 and 12 months after TUSP was higher in the older age group than in the younger age group. The change in PVR was consistently more remarkable in the older age group than in the younger age group. The change in Qmax at 12 months postoperatively was greater in the lower age group than in the higher age group (Figure 4A-4D).

\section{Discussion}

In this multicenter retrospective study, we comparatively evaluated BPH patients treated with TUSP. The overall outcome of TUSP in all patients was significant, as evidenced by a low rate of severe intraoperative and postoperative complications and a shorter operative time. In this study, the effectiveness of TUSP in treating different BPH groups was compared and analyzed, focusing on a prostate volume of $50 \mathrm{~mL}$ and age 70 as the cut-off values. The results showed that TUSP was more effective overall in $\mathrm{BPH}$ patients with a prostate volume less than $50 \mathrm{~mL}$ and younger than 70 years.

More attention should be paid to the QoL of older men than just those with fatal malignant diseases. $\mathrm{BPH}$ is a benign lesion, and lower urinary tract obstructions tend to have a longer course, so BPH is not usually life-threatening. The most significant distress it poses to patients is the dramatically impaired QoL. As medical care improves and the average human lifespan is extended globally, the phenomenon of aging is becoming more prominent. As a result, the growing incidence of $\mathrm{BPH}$ has made $\mathrm{BPH}$-induced LUTS an increasingly important public health problem affecting 
the QoL of older men (23). BPH is a histological diagnosis characterized by an abnormal proliferation of glandular cells, mainly prostate epithelial and stromal cells. The hyperplastic prostate may cause mechanical obstruction of the urethra and may also alter the tension of the smooth muscle of the urethra, which in combination contributes to dysuria. LUTS causes severe discomfort to the patient. The main effect of the drug regimen for $\mathrm{BPH}$ is to mitigate the progression of the disease. However, it is difficult to reverse the disease or achieve a satisfactory outcome. Surgery is an effective treatment for BPH. In this study, within 1 year of TUSP, patients had significantly lower IPSS and QoL scores, less PVR, and a significantly larger Qmax than before surgery, suggesting that TUSP has a favorable therapeutic effect.

TUSP is a modification of the TUDP procedure, with similar principles but a differently designed instrument. For TUDP, the spherical balloon primarily dilates the proximal end of the prostatic urethra (18). In contrast, the double-column balloon catheter used in TUSP, with both proximal and distal balloons working simultaneously, has dilating effects on the bladder neck, prostatic urethra, and the membranous urethra (24). TUSP reduces urethral closure pressure by dilating the prostatic capsule through the apex position of the prostate via the internal balloon. The external balloon expands and eventually creates a wide channel, reducing tension on the urethral wall. As the patient recovers, surrounding tissues such as retropubic fat and fascial tissue fill the perineal fissure, and the urethral tissue swells, causing the prostate to shift. In contrast, the distal balloon dilates the membranous urethra, and the external sphincter displaces the prostate tip, promoting long-term patency of the urethra (18).

The rich blood flow in the prostate gland and the high intraoperative bleeding are risks of conventional TURP (25). This study found that blood loss after TUSP was significantly lower in BPH patients with a prostate volume less than $50 \mathrm{~mL}$ than those with more than $50 \mathrm{~mL}$. This result might be related to the richer blood flow to the larger prostate. There was more bleeding in the older age group than in the younger age group, which might be caused by the higher vascular fragility in older patients (26). However, the exact mechanism needs to be further elucidated. In addition to more blood loss, the traditional TURP has several other shortcomings. TURP has a long procedure time, averaging around 62-83 minutes (27). This study showed that the average time for TUSP was less than 30 minutes, so patients tolerated TUSP better. Excessive absorption of the irrigating fluid during TURP may cause dilutional hyponatremia and the occurrence of TURP syndrome (28). Other postoperative complications include urinary incontinence, retrograde ejaculation, BNC, and urethral stricture. Patients with low surgical risk are suitable for TURP, while patients with high surgical risk or who cannot tolerate general anesthesia are more suitable for a less invasive procedure. These shortcomings have led to a continuing search for less invasive treatments. The treatment of BPH patients with TUDP was first reported by Castaneda et al. (29). Compared to TURP, TUDP is a relatively simple procedure and less traumatic to the prostate organ. However, as its clinical application has increased, TUDP has revealed some drawbacks. One clinical study reported that a significant number of patients had a recurrence of urethral obstruction or other complications within 1 year of TUDP, and therefore its clinical use has been gradually declining (30). In brief, compared with TURP, TUSP has the advantages of low risk of bleeding, short procedure time, low incidence of dilutional hyponatremia and TURP syndrome, and favorable protection of sexual function. However, the recurrence rate of urethral obstruction after TUSP treatment is higher than that of TURP.

TUSP causes no incision or damage to the prostate organ, only dilating the urethral prostatic obstruction, making the procedure less invasive and relatively simple to perform without damaging the nerves and urethral sphincter (1). Intraoperative thermal damage to the bladder neck can cause difficulty in urination after surgery and is one of the main concerns affecting patients' QoL and satisfaction with prostate enlargement (31). TUSP enhances the intraoperative protection of the bladder neck. It is noteworthy that there is no clear correlation between prostate volume and LUTS severity (32). The causes of LUTS may be varied and may be obstructive, irritating, or a combination of factors (33). Hyperplasia of the periurethral prostatic glands and migratory zone components is the leading cause of LUTS and depends on the bioavailability of testosterone and dihydrotestosterone. Prostate fibrosis and smooth muscle contraction are predominantly responsible for LUTS when the prostate size is relatively small (34). Chronic prostatitis is a fundamental cause of prostate fibrosis. In small volume BPH combined with chronic prostatitis and fibrotic changes, thermal damage from TURP may cause additional fibrosis and is highly correlated with BNC (35). The laser may produce thermal damage in laser-based BPH surgery and lead to a new fibrotic pathology, increasing the post-treatment BNC risk. Furthermore, TUSP facilitates the protection of sexual 
function and is more appropriate for elderly patients with various underlying diseases and poor surgical tolerance (19).

This study found that the changes in IPSS, PVR, and Qmax were more significant in the small volume prostate group than in the large volume prostate group at the 12-month follow-up after TUSP. This may be due to the smaller prostate displacement during TUSP in patients with small volume prostate $\mathrm{BPH}$, which resulted in more significant postoperative symptom changes. In the large volume prostate $\mathrm{BPH}$ group, the prostate may still have some compression of the urethra after TUSP. The micturition urgency and transient incontinence rate after TUSP were lower in the small volume prostate group than in the large volume group. This may be because the structural changes in a small prostate are less substantial than in a large prostate, and therefore, the displacement of the prostate by TUSP is smaller.

In this study, TUSP was more effective in patients under 70 years of age than in patients over 70 years of age. As previously mentioned, the mean prostate volume was significantly higher in patients over 70 years of age than in patients under 70 years of age. The larger prostate volume may be the main reason why TUSP is slightly less effective in these older patients. In addition, many factors can influence the progression of $\mathrm{BPH}$. In $\mathrm{BPH}$, inflammatory cell infiltration is often observed in the prostate (36). A previous study has shown an association between recurrent prostatitis and an increased risk of acute urinary retention, suggesting that chronic inflammation may play a role in the development of $\mathrm{BPH}$ (37). Many patients with BPH experience chronic prostatitis and may develop fibrosis as a result. Fibrosis is an abnormal form of tissue repair that involves the accumulation of myofibroblasts, collagen deposition, extracellular matrix remodeling, and tissue stiffening (20). Like other soft tissues, the prostate is susceptible to the fibrotic changes associated with inflammation and aging, producing a stiffer tissue structure that can negatively affect the urethral function and promote symptoms of urinary tract obstruction (38). The long duration of prostate inflammation in older patients may intensify fibrosis in the bladder neck or prostate, resulting in poorer outcomes after TUSP than in younger patients. However, there is a lack of evidence, and more research is needed. In addition, the need to protect sexual function in younger patients is critical, and therefore the less-invasive nature of TUSP makes it beneficial and suitable for younger patients (39).

Notably, TUSP has some limitations. Firstly, in a few cases, TUSP may cause laceration of the prostatic urethra and consequential bleeding. Secondly, TUSP devices are not yet equipped with visualization devices. As a result, the operation is somewhat challenging for surgeons inexperienced in the procedure. Furthermore, a pathological biopsy of prostate tissue excised by TURP might assist in the early diagnosis of incidental prostate cancer. In contrast, this advantage is not available with TUSP as there is no excised tissue.

Based on this study, we recommend a careful evaluation when selecting surgical options for patients with BPH. Factors to be evaluated include prostate volume, patient age and surgical tolerance of the patient. For BPH patients with advanced age or small prostate volume, TUSP may be considered as a priority treatment. Long-term followup after TUSP needs to be emphasized to provide early intervention for recurrent voiding problems when necessary.

It should be noted that this study has some limitations. Firstly, the follow-up period for all patients was only 12 months. A more extended follow-up period is warranted to validate the findings of this study. Second, not all outcomes were reported for each included observation, and there were some missing data, which may affect the accuracy of the results. In addition, although $\mathrm{BPH}$ is the leading cause of LUTS in older men, other physical problems may also contribute to LUTS. For example, congestive heart failure may result in significant nocturia due to nocturnal lower limb edema activity. Diabetes mellitus may cause frequent urination due to diuresis and irritation due to autonomic bladder neuropathy (40). These factors may influence the results of this study. Nevertheless, this multicenter retrospective study still provided a valuable comparative assessment to confirm the efficacy of TUSP in treating BPH and identify a more specific TUSP-suitable population.

\section{Conclusions}

TUSP has the advantage of resolving urinary problems without excision of the prostate, is minimally invasive, and has a short procedure time. In this study, TUSP was shown to be more effective in younger patients presenting with $\mathrm{BPH}$ or those with small prostate volumes. It is an appropriate treatment for patients with $\mathrm{BPH}$ who have poor surgical tolerance. A larger cohort and longer follow-up are necessary to substantiate these findings further.

\section{Acknowledgments}

Funding: This project has been funded by the National 
Natural Science Foundation of China (No. 81871153 to Jiaming Wen and No. 81970601 to Bohan Wang).

\section{Footnote}

Reporting Checklist: The authors have completed the STROBE reporting checklist. Available at https://tau. amegroups.com/article/view/10.21037/tau-21-1138/rc

Data Sharing Statement: Available at https://tau.amegroups. com/article/view/10.21037/tau-21-1138/dss

Conflicts of Interest: All authors have completed the ICMJE uniform disclosure form (available at https://tau.amegroups. com/article/view/10.21037/tau-21-1138/coif). The authors have no conflicts of interest to declare.

Ethical Statement: The authors are accountable for all aspects of the work in ensuring that questions related to the accuracy or integrity of any part of the work are appropriately investigated and resolved. All procedures performed in studies involving human participants were in accordance with the ethical standards of the institutional and/or national research committee(s) and with the Helsinki Declaration (as revised in 2013). The study was approved by institutional ethics board of The Second Affiliated Hospital, School of Medicine, Zhejiang University (No. 2021-1062). Written informed consents were obtained from all patients.

Open Access Statement: This is an Open Access article distributed in accordance with the Creative Commons Attribution-NonCommercial-NoDerivs 4.0 International License (CC BY-NC-ND 4.0), which permits the noncommercial replication and distribution of the article with the strict proviso that no changes or edits are made and the original work is properly cited (including links to both the formal publication through the relevant DOI and the license). See: https://creativecommons.org/licenses/by-nc-nd/4.0/.

\section{References}

1. Huang Y, Li J, Yang S, et al. Efficacy and safety of transurethral split of prostate for benign prostatic hyperplasia: a meta-analysis. BMC Urol 2020;20:141.

2. Chughtai B, Forde JC, Thomas DD, et al. Benign prostatic hyperplasia. Nat Rev Dis Primers 2016;2:16031.

3. Rule AD, Lieber MM, Jacobsen SJ. Is benign prostatic hyperplasia a risk factor for chronic renal failure? J Urol 2005;173:691-6.

4. Foo KT. What is a disease? What is the disease clinical benign prostatic hyperplasia (BPH)? World J Urol 2019;37:1293-6.

5. Gratzke C, Bachmann A, Descazeaud A, et al. EAU Guidelines on the Assessment of Non-neurogenic Male Lower Urinary Tract Symptoms including Benign Prostatic Obstruction. Eur Urol 2015;67:1099-109.

6. Rassweiler J, Teber D, Kuntz R, et al. Complications of transurethral resection of the prostate (TURP)--incidence, management, and prevention. Eur Urol 2006;50:969-79; discussion 980.

7. Sturch P, Woo HH, McNicholas T, et al. Ejaculatory dysfunction after treatment for lower urinary tract symptoms: retrograde ejaculation or retrograde thinking? BJU Int 2015;115:186-7.

8. Huang SW, Tsai CY, Tseng CS, et al. Comparative efficacy and safety of new surgical treatments for benign prostatic hyperplasia: systematic review and network meta-analysis. BMJ 2019;367:15919.

9. Chen YZ, Lin WR, Chow YC, et al. Analysis of risk factors of bladder neck contracture following transurethral surgery of prostate. BMC Urol 2021;21:59.

10. Gilling P. TURP remains a safe and effective alternative for benign prostatic hyperplasia (BPH) surgery. BJU Int 2014;113:5-6.

11. Shvero A, Kloniecke E, Capella C, et al. HoLEP techniques - lessons learned. Can J Urol 2021;28:11-6.

12. Gravas S, Cornu J, Gacci M, et al. EAU Guidelines: Treatment of non-neurogenic male LUTS. 2019. Available online: https://uroweb.org/guideline/treatment-of-nonneurogenic-male-luts/

13. Becker B, Gross AJ, Netsch C. Safety and efficacy using a low-powered holmium laser for enucleation of the prostate (HoLEP): 12-month results from a prospective low-power HoLEP series. World J Urol 2018;36:441-7.

14. Dornbier R, Pahouja G, Branch J, et al. The New American Urological Association Benign Prostatic Hyperplasia Clinical Guidelines: 2019 Update. Curr Urol Rep 2020;21:32.

15. Peng B, Wang GC, Zheng JH, et al. A comparative study of thulium laser resection of the prostate and bipolar transurethral plasmakinetic prostatectomy for treating benign prostatic hyperplasia. BJU Int 2013;111:633-7.

16. Li Q, Zhang J, Cao Y, et al. Observation on the effect 
of transurethral plasmakinetic enucleation of prostate for 126 patients with large-volume prostatic hyperplasia. Chinese Journal of Primary Medicine and Pharmacy 2020;27:2627-31.

17. Chang Y, Chang J, Wang H. Transurethral balloon dilatation of the Prostate and Transurethral Plasmakinetic resection of the Prostate in the treatment of Prostatic Hyperplasia. Pak J Med Sci 2018;34:736-9.

18. Huang W, Huang Z, Xiao G, et al. Effect of transurethral split of the prostate using a double-columnar balloon catheter for benign prostatic hyperplasia: A singlecenter experience of 565 consecutive patients. Medicine (Baltimore) 2016;95:e4657.

19. Huang W, Guo Y, Xiao G, et al. Treatment of benign prostatic hyperplasia using transurethral split of the prostate with a columnar balloon catheter. J Endourol 2015;29:344-50.

20. Ma J, Gharaee-Kermani M, Kunju L, et al. Prostatic fibrosis is associated with lower urinary tract symptoms. J Urol 2012;188:1375-81.

21. Wang B, Zhang S, Sun C, et al. Comparison between a transurethral prostate split and transurethral prostate resection for benign prostatic hyperplasia treatment in a small prostate volume: a prospective controlled study. Ann Transl Med 2020;8:1016.

22. Tsunemori H, Sugimoto M. Effects of inflammatory prostatitis on the development and progression of benign prostatic hyperplasia: A literature review. Int J Urol 2021;28:1086-92.

23. Roehrborn CG. Current medical therapies for men with lower urinary tract symptoms and benign prostatic hyperplasia: achievements and limitations. Rev Urol 2008;10:14-25.

24. Kaiho Y, Nakagawa H, Saito H, et al. Nerves at the ventral prostatic capsule contribute to erectile function: initial electrophysiological assessment in humans. Eur Urol 2009;55:148-54.

25. Rahman MM, Johura FT, Rasul MA, et al. Effect of Preoperative Dutasteride on Bleeding Related to Transurethral Resection of Prostate in Patients with Benign Prostatic Hyperplasia. Journal of Biosciences and Medicines 2019;7:157.

26. Ungvari Z, Yabluchanskiy A, Tarantini S, et al. Repeated Valsalva maneuvers promote symptomatic manifestations of cerebral microhemorrhages: implications for the pathogenesis of vascular cognitive impairment in older adults. Geroscience 2018;40:485-96.
27. Jiang YL, Qian LJ. Transurethral resection of the prostate versus prostatic artery embolization in the treatment of benign prostatic hyperplasia: a meta-analysis. BMC Urol 2019;19:11.

28. HAZARIKA PC. TURP Syndrome-A Quick Review and Update. Indian Journal of Clinical Practice 2020;31:224-8.

29. Castaneda F, Letourneau JG, Reddy P, et al. Alternative treatment of prostatic urethral obstruction secondary to benign prostatic hypertrophy. Nonsurgical balloon catheter prostatic dilatation. Rofo 1987;147:426-9.

30. Vale JA, Miller PD, Kirby RS. Balloon dilatation of the prostate--should it have a place in the urologist's armamentarium? J R Soc Med 1993;86:83-6.

31. Browne BM, Vanni AJ. Management of Urethral Stricture and Bladder Neck Contracture Following Primary and Salvage Treatment of Prostate Cancer. Curr Urol Rep 2017;18:76.

32. Simon RM, Howard LE, Moreira DM, et al. Does Prostate Size Predict the Development of Incident Lower Urinary Tract Symptoms in Men with Mild to No Current Symptoms? Results from the REDUCE Trial. Eur Urol 2016;69:885-91.

33. Langan RC. Benign Prostatic Hyperplasia. Prim Care 2019;46:223-32.

34. Kang M, Kim M, Choo MS, et al. Urodynamic Features and Significant Predictors of Bladder Outlet Obstruction in Patients With Lower Urinary Tract Symptoms/Benign Prostatic Hyperplasia and Small Prostate Volume. Urology 2016;89:96-102.

35. Maddox M, Pareek G, Al Ekish S, et al. Histopathologic changes after bipolar resection of the prostate: depth of penetration of bipolar thermal injury. J Endourol 2012;26:1367-71.

36. De Nunzio C, Presicce F, Tubaro A. Inflammatory mediators in the development and progression of benign prostatic hyperplasia. Nat Rev Urol 2016;13:613-26.

37. Lloyd GL, Ricke WA, McVary KT. Inflammation, Voiding and Benign Prostatic Hyperplasia Progression. J Urol 2019;201:868-70.

38. Sampson N, Berger P, Zenzmaier C. Therapeutic targeting of redox signaling in myofibroblast differentiation and age-related fibrotic disease. Oxid Med Cell Longev 2012;2012:458276.

39. Mónica FZ, De Nucci G. Tadalafil for the treatment of 
benign prostatic hyperplasia. Expert Opin Pharmacother 2019;20:929-37.

40. Kim EH, Larson JA, Andriole GL. Management of Benign

Cite this article as: Wei J, Zhang S, Wang B, Ke M, Liu S, Yang Z, Zhou G, Qian J, Lv W, Fan Y, Shi Z, Wan L, Chen Y, He J, Liang H, Long H, Wang S, Wang H, Chen B, Shao H, Yang B, Sun C, Huangfu Q, Du C, Cai M, Wen J. A multicenter retrospective study of transurethral prostate split for benign prostate hyperplasia. Transl Androl Urol 2022;11(2):213-227. doi: $10.21037 /$ tau-21-1138
Prostatic Hyperplasia. Annu Rev Med 2016;67:137-51.

(English Language Editor: D. Fitzgerald) 